\title{
The effects of Lycii Radicis Cortex on RANKL-induced osteoclast differentiation and activation in RAW 264.7 cells
}

\author{
JAE-HYUN KIM ${ }^{1}$, EUN-YOUNG KIM ${ }^{1}$, BINA LEE ${ }^{1}$, JU-HEE MIN ${ }^{1}$, DEA-UK SONG ${ }^{1}$, JEONG-MIN LIM ${ }^{1}$, \\ JI WHAN EOM ${ }^{1}$, MIJUNG YEOM ${ }^{2}$, HYUK-SANG JUNG ${ }^{1}$ and YOUNGJOO SOHN ${ }^{1}$ \\ ${ }^{1}$ Department of Anatomy, ${ }^{2}$ Acupuncture and Meridian Science Research Center, \\ College of Korean Medicine, Kyung Hee University, Seoul 130-701, Republic of Korea
}

Received July 21, 2015; Accepted January 25, 2016

DOI: $10.3892 /$ ijmm.2016.2477

\begin{abstract}
Post-menopausal osteoporosis is a serious age-related disease. After the menopause, estrogen deficiency is common, and excessive osteoclast activity causes osteoporosis. Osteoclasts are multinucleated cells generated from the differentiation of monocyte/macrophage precursor cells such as RAW 264.7 cells. The water extract of Lycii Radicis Cortex (LRC) is made from the dried root bark of Lycium chinense Mill. and is termed 'Jigolpi' in Korea. Its effects on osteoclastogenesis and post-menopausal osteoporosis had not previously been tested. In the present study, the effect of LRC on receptor activator of nuclear factor- $\mathrm{\kappa B}(\mathrm{NF}-\mathrm{\kappa B})$ ligand (RANKL)-induced osteoclast differentiation was demonstrated using a tartrate-resistant acid phosphatase (TRAP) assay and pit formation assay. Moreover, in order to analyze molecular mechanisms, we studied osteoclastogenesis-related markers such as nuclear factor of activated T-cells cytoplasmic 1 (NFATc1), c-Fos, receptor activator of NF- $\kappa B$ (RANK), TRAP, cathepsin K (CTK), matrix metallopeptidase-9 (MMP-9), calcitonin receptor (CTR) and carbonic anhydrase II (CAII) using RT-qPCR and western blot analysis. Additionally, we also determined the effect of LRC on an ovariectomized (OVX) rat model. We noted that LRC inhibited RANKL-induced osteoclast differentiation via suppressing osteoclastogenesis-related markers. It also inhibited osteoporosis in the OVX rat model by decreasing loss of bone density and trabecular area. These results suggest that LRC exerts a positive effect on menopausal osteoporosis.
\end{abstract}

Correspondence to: Professor Youngjoo Sohn, Department of Anatomy, College of Korean Medicine, Kyung Hee University, 26 Kyungheedae-ro, Dongdaemun-gu, Seoul 130-701, Republic of Korea

E-mail: youngjoos@khu.ac.kr

Key words: osteoclast, receptor activator of nuclear factor- $\kappa \mathrm{B}$ ligand, Lycii Radicis Cortex, nuclear factor of activated T-cells cytoplasmic 1, c-Fos, ovariectomy

\section{Introduction}

Osteoporosis is a serious health problem that is related to aging; it is characterized by decreased bone density and increased risk of fracture (1). Bone remodeling is a continuous process between bone resorption (activity of osteoclasts) and formation (activity of osteoblasts). Disintegration due to these opposing processes may cause bone diseases (2-4). Osteoclasts are activated for many reasons, one of which is the imbalance of hormones caused by the menopause (5). The absence of estrogen, induced by the menopause, increases the formation and the activity of osteoclasts, which play key roles in bone loss, and osteoclasts ultimately increase the risk of menopausal osteoporosis (6). Therefore, inhibiting osteoclast formation and function is an important therapeutic strategy.

Osteoclasts are multinucleated cells generated from monocyte/macrophage precursor cells, and osteoclast formation requires receptor activator of nuclear factor- $\kappa \mathrm{B}(\mathrm{NF}-\kappa \mathrm{B})$ ligand (RANKL). RAW 264.7 cells have been demonstrated to play an important role, using in vitro studies, on osteoclast formation and function (7). Adding RANKL to RAW 264.7 cells induces osteoclast differentiation (4). The receptor activator of NF- $\mathrm{KB}$ (RANK) is expressed on RAW 264.7 cell surfaces and conjugates with RANKL, which is essential for osteoclastogenesis (8). RANKL to RANK interaction activates tumor necrosis factor receptor-associated factor 6 (TRAF6), and TRAF6 then activates c-Fos, as an important transcription factor for osteoclastogenesis, and downregulates osteoclastogenesis via nuclear factor of activated T-cells cytoplasmic 1 (NFATc1) activation (9). As a master transcription factor of osteoclastogenesis, NFATc1 regulates diverse osteoclastogenesis-related genes such as tartrate-resistant acid phosphatase (TRAP), cathepsin $\mathrm{K}$ (CTK), calcitonin receptor (CTR) and matrix metallopeptidase-9 (MMP-9) (4,10-12).

Various therapies are available for post-menopausal osteoporosis, such as estrogen replacement therapy, bisphosphonate and calcitonin, and it has previously been noted that estrogen replacement therapy is commonly used in cases of post-menopausal osteoporosis $(3,13)$. However, long-term estrogen replacement therapy has been demonstrated to cause various side-effects such as breast cancer and endometrial cancer $(3,14)$. An alternative therapy which is thus worthy of 
consideration is natural herbs, which exert positive effects on osteoporosis and have fewer harmful side-effects.

Lycii Radicis Cortex (LRC) is the dried root bark of Lycium chinense Mill. and is termed 'Jigolpi' in Korea. Traditionally, LRC has been used to treat lung fever and reduce fever of the blood, lower blood pressure, decrease blood sugar, and, particularly, decrease steaming bone disorder $(15,16)$. LRC has been reported to perform various biological roles, and acts as an anti-inflammatory (17), anti-oxidant (18), anti-depressant (19), tumor growth inhibitor (20) and blood glucose regulator (21). However, the inhibitory effect of LRC on osteoclastogenesis has not been previously investigated, to the best of our knowledge.

In the present study, we aimed to investigate the effect of LRC on osteoclastogenesis, bone resorption activity and expression levels of osteoclastic markers in RAW 264.7 cells and on post-menopausal osteoporosis induced by ovariectomy in ovariectomized (OVX) rats.

\section{Materials and methods}

Reagents. RANKL was purchased from PeproTech (London, UK). Dulbecco's modified Eagle's medium (DMEM) was purchased from Welgene (Daejeon, Korea). Cell medium minimum essential medium- $\alpha(\alpha-\mathrm{MEM})$ and fetal bovine serum (FBS) were both purchased from Gibco (Gaithersburg, NY, USA). Penicillin/streptomycin was purchased from Invitrogen (Carlsbad,CA, USA). Dulbecco's phosphate-buffered saline (DPBS) was obtained from Gibco. An aqueous nonradioactive cell proliferation kit (for MTS assay) was purchased from Promega (Madison, WI, USA). A TRAP staining kit was purchased from Sigma-Aldrich (St. Louis, MI, USA). An Osteo Assay Stripwell plate was purchased from Corning Inc. (New York, NY, USA). We obtained the reverse transcription kit from Invitrogen. Taq polymerase was obtained from Kapa Biosystems (Woburn, MA, USA). PCR primers were from Genotech (Daejeon, Korea). Primary antibodies against c-Fos (Cat. no. sc-447) and $\beta$-actin (Cat. no. sc-8432) were purchased from Santa Cruz Biotechnology, Inc. (Santa Cruz, CA, USA) and NFATc1 antibody (Cat. no. 556602) was obtained from BD Pharmingen (San Diego, CA, USA). Peroxidase IgG secondary antibody (Cat. no. 115-035-062) was purchased from Jackson ImmunoResearch (West Grove, PA, USA). Protease inhibitor cocktail and phosphatase inhibitor cocktail were both purchased from Sigma-Aldrich.

Sample preparation. LRC was purchased from Kyung Hee University Medical Center. The extract was prepared by decocting $300 \mathrm{~g}$ of the dried herb using 3 liters of boiling distilled water for $2 \mathrm{~h}$ and filtered using filter paper (no. 3; Whatman, Maidstone, UK). The extract was concentrated using a rotary evaporator (Eyela, Tokyo, Japan), lyophilized, and it yielded $11.1 \mathrm{~g}$ dried powder (yield ratio $3.7 \%$ ), and the extract was then stored at $-20^{\circ} \mathrm{C}$ until use.

Cell culture and cytotoxicity assay. Murine macrophage RAW 264.7 cells were cultured in DMEM with 10\% FBS and $1 \%$ penicillin/streptomycin at $37^{\circ} \mathrm{C}$ in a humidified atmosphere of $5 \% \mathrm{CO}_{2}$ in air. For the cytoxicity assay, RAW 264.7 cells were cultured in DMEM with $10 \%$ FBS and $1 \%$ penicillin/streptomycin and plated in a 96 -well plate at $5 \times 10^{3}$ cells/well. After
$24 \mathrm{~h}$, various concentrations of LRC were added to the medium for $24 \mathrm{~h}$. MTS solution was added at $20 \mu \mathrm{l} /$ well. The plate was incubated for $2 \mathrm{~h}$ at $37^{\circ} \mathrm{C}$. Cell viability was measured using an enzyme-linked immunosorbent assay (ELISA reader; Molecular Devices, Sunnyvale, CA, USA) at $562 \mathrm{~nm}$ optical density.

TRAP staining and the TRAP activity assay. In order to study osteoclast differentiation, RAW 264.7 cells were cultured in $\alpha$-MEM with $10 \%$ FBS and $1 \%$ penicillin/streptomycin in a 96 -well plate $5 \times 10^{3}$ cells/well. After $24 \mathrm{~h}$, the $\alpha$-MEM was changed for $10 \% \mathrm{FBS}$ and $1 \%$ penicillin/streptomycin. RANKL $(100 \mathrm{ng} / \mathrm{ml})$ and various concentrations of LRC were added to the media for 5 days. The medium was changed every 2 days. After 5 days, multinucleated osteoclasts were observed. Mature osteoclasts were washed with DPBS and fixed in $10 \%$ formaldehyde for $10 \mathrm{~min}$. After being fixed, cells were stained using a TRAP staining kit (Sigma-Aldrich) according to the manufacturer's instructions. Cells were washed with deionized water. We then counted TRAP-positive multinucleated ( $>3$ nuclei) under an inverted microscope (Olympus, Tokyo, Japan). In order to measure TRAP activity, we added $50 \mu \mathrm{l}$ supernatant to a 96-well plate, dissolved $4.93 \mathrm{mg}$ Pnpp in $850 \mu 10.5 \mathrm{M}$ acetate, mixed it with $150 \mu \mathrm{l}$ tartrate acid solution and separated it (50 $\mu \mathrm{l}$ each) into 96-well plates. After $30 \mathrm{~min}$, we added $50 \mu \mathrm{l} 0.5 \mathrm{M} \mathrm{NaOH}$ and measured optical density at $405 \mathrm{~nm}$ using an ELISA reader.

Pitformation assay. RAW 264.7 cells were cultured in $\alpha$-MEM with $10 \%$ FBS and $1 \%$ penicillin/streptomycin and plated in an Osteo Assay Stripwell plate at $5 \times 10^{3}$ cells/well. After $24 \mathrm{~h}$, $\alpha$-MEM was changed for $10 \%$ FBS and $1 \%$ penicillin/streptomycin. RANKL (100 ng/ml) and various concentrations of LRC were added to the medium for 5 days. The medium was changed every 2 days. After 5 days, multinucleated osteoclasts were observed. The mature osteoclasts were then lysed by $4 \% \mathrm{NaClO}$ and lysates were washed with deionized water. The pit area in each plate was studied and measured under an inverted microscope.

Reverse transcription-quantitative polymerase chain reaction (RT-qPCR). In order to generate osteoclasts, RAW 264.7 cells were cultured with $\alpha$-MEM, $10 \%$ FBS and $1 \%$ penicillin/ streptomycin. RANKL (100 ng/ml) was added for 4 days in the presence of various concentrations of LRC. Total RNA was prepared using TRIzol (Takara Bio, Otsu, Japan) according to the manufacturer's instructions. The concentration of total RNA was determined (absorbance at 260 and $280 \mathrm{~nm}$ ) with a NanoDrop 2.0 spectrophotometer (Thermo Fisher Scientific, Pittsburgh, PA, USA), and $2 \mu \mathrm{g}$ total RNA was generated using a reverse transcription kit (Invitrogen) according to the manufacturer's instructions. The PCR cycles were as follows: $22-40$ cycles of $1 \mathrm{~min}$ at $94^{\circ} \mathrm{C}$ (denaturation), $1 \mathrm{~min}$ at $55-58^{\circ} \mathrm{C}$ (annealing), and a $1-\min 72^{\circ} \mathrm{C}$ (extension), using Taq polymerase. Primer sequences are shown in Table I. The cDNA samples after reaction were separated on a 1-1.2\% agarose gel, stained with SYBR-Green (Invitrogen) and studied using ImageJ software.

Western blot analysis. The cells incubated with various concentrations of $\operatorname{LRC}(1,10$ and $100 \mu \mathrm{g} / \mathrm{ml})$ were washed with cold DPBS, and cells were lysed in lysis buffer $(50 \mathrm{mM}$ 
Table I. Primer sequences for RT-qPCR.

\begin{tabular}{|c|c|c|c|}
\hline Target & Primer sequences $\left(5^{\prime} \rightarrow 3^{\prime}\right)$ & Annealing temperature $\left({ }^{\circ} \mathrm{C}\right)$ & Cycle \\
\hline$N F A T c 1$ & $\begin{array}{l}\text { F: 5'-TGC TCC TCC TCC TGC TGC TC-3' } \\
\text { R: 5'-CGT CTT CCA CCT CCA CGT CG-3' }\end{array}$ & 58 & 32 \\
\hline$c-F o s$ & $\begin{array}{l}\text { F: 5'-ATG GGC TCT CCT GTC AAC AC-3' } \\
\text { R: 5'-GGC TGC CAA AAT AAA CTC CA-3' }\end{array}$ & 58 & 33 \\
\hline$R A N K$ & $\begin{array}{l}\text { F: 5'-AAA CCT TGG ACC AAC TGC AC-3' } \\
\text { R: 5'-ACC ATC TTC TCC TCC CHA GT-3' }\end{array}$ & 53 & 32 \\
\hline TRAP & $\begin{array}{l}\text { F: } 5 \text { '-ACT TCC CCA GCC CTT ACT ACC G-3' } \\
\text { R: 5'-TCA GCA CAT AGC CCA CAC CG-3' }\end{array}$ & 58 & 30 \\
\hline CTK & $\begin{array}{l}\text { F: 5'-AGG CGG CTA TAT GAC CAC TG-3' } \\
\text { R: 5'-CCG AGC CAA GAG AGC ATA TC-3' }\end{array}$ & 58 & 27 \\
\hline CTR & $\begin{array}{l}\text { F: } 5 \text { '-TGC ATT CCC GGG ATA CAC AG-3' } \\
\text { R: 5'-AGG AAC GCA GAC TTC ACT GG-3' }\end{array}$ & 59 & 40 \\
\hline$M M P-9$ & $\begin{array}{l}\text { F: 5'-CGA CTT TTG TGG TCT TCC CC-3' } \\
\text { R: 5'-TGA AGG TTT GGA ATC GAC CC-3' }\end{array}$ & 58 & 33 \\
\hline CAII & $\begin{array}{l}\text { F: 5'-CTC TCA GGA CAA TGC AGT GCT GA-3' } \\
\text { R: 5'-ATC CAG GTC ACA CAT TCC AGC A-3' }\end{array}$ & 58 & 32 \\
\hline GAPDH & $\begin{array}{l}\text { F: } 5 \text { '-ACT TTG TCA AGC TCA TTT CC-3' } \\
\text { R: 5'-TGC AGC GAA CTT TAT TGA TG-3' }\end{array}$ & 58 & 30 \\
\hline
\end{tabular}

NFATc1, nuclear factor of activated T-cells cytoplasmic 1; RANK, receptor activator of nuclear factor- $\mathrm{B}$; TRAP, tartrate-resistant acid phosphatase; CTK, cathepsin K; CTR, calcitonin receptor; MMP-9, matrix metallopeptidase-9, CAII, carbonic anhydrase II.

Tris-Cl, $150 \mathrm{mM} \mathrm{NaCl}, 1 \% \mathrm{NP}-40,0.5 \%$ Na-deoxycholate, $0.1 \%$ SDS, protease inhibitor cocktail, phosphatase inhibitor cocktail) and incubated on ice for $30 \mathrm{~min}$. After centrifugation at $13,200 \mathrm{rpm}$ for $20 \mathrm{~min}$ at $4^{\circ} \mathrm{C}$, the supernatants were stored at $-70^{\circ} \mathrm{C}$ until use. Protein concentration was calculated using a BCA protein assay kit (Thermo Fisher Scientific). The protein samples $(30 \mu \mathrm{g})$ were separated by $10-15 \%$ SDS-PAGE and transferred to a nitrocellulose membrane (Whatman, Dassel, Germany). We then blocked them with 5\% skimmed milk for $1 \mathrm{~h}$, and the membrane was then incubated with primary antibodies, namely NFATc1, c-Fos and actin, in 1\% BSA solution at $4^{\circ} \mathrm{C}$ overnight. Subsequently, the membrane was probed with the secondary antibody. The protein was detected using ECL solution (Santa Cruz Biotechnology, Inc.).

Animal model of OVX rats and histopathological examination. The animal experiments were conducted in compliance with the principles of the Institutional Animal Care and Use Committee of Kyung Hee University Laboratory Animal Center: the permission no. is KHUASP (SE)-13-051. Sprague-Dawley (SD) rats were purchased from Nara Biotech (Seoul, Korea). The $17 \beta$-estradiol $\left(E_{2}\right)$ was from Sigma-Aldrich. Twelve-week-old female Sprague-Dawley rats weighing $240-250 \mathrm{~g}$ were used. Rats were housed at $22 \pm 1^{\circ} \mathrm{C}$ in an atmosphere with $55 \pm 10 \%$ humidity on a $12 \mathrm{~h}$ light/dark cycle with free access to food and water. After acclimatization to the laboratory environment for 1 week, rats were divided into 5 groups (8 rats/group), i) sham-operated rats, ii) OVX rats, iii) OVX rats which received $17 \beta$-estradiol $(100 \mu \mathrm{g} / \mathrm{kg}$ p.o.), iv) OVX rats treated with low doses of LRC $(5 \mathrm{mg} / \mathrm{kg}$ p.o.), and v) OVX rats treated with high doses of LRC (50 mg/ $\mathrm{kg}$ p.o.).

After the operation, sham-operated and OVX rats were administrated distilled water orally. After the ovariectomy, the rats were treated 5 times/week for 8 weeks and weighed each week. At the end of the treatment, the rats were euthanized while under deep anesthesia with high doses of pentobarbital sodium (80 mg/kg) and blood samples were acquired by cardiac puncture for biochemical analyses. The uteri and both sides of the femurs were dissected out and immediately weighed on an electronic scale. The femurs were then fixed in $10 \%$ neutral buffered formalin $(\mathrm{NBF})$ for 2 days and decalcified using $10 \%$ ethylenediaminetetraacetate (EDTA) for 3 weeks. The samples, which were cut into $5-\mu \mathrm{m}$ thick sections using a microtome (Zeiss, Oberkochen, Germany), were embedded in paraffin. Tissues were deparaffinized, rehydrated, and stained with hematoxylin and eosin (H\&E). Histopathological changes were observed using light microscopy (at x40 and x100 magnification).

Measurement of bone density after inducing OVX. At the end of treatment, the rats were sacrificed and the bone density of the right femurs was measured using Archimedes' principle, as previously described (22). Right femurs were briefly placed in vials filled with deionized water. The vials were placed in a vacuum for 90 min to ensure that all trapped air diffused from the bones. Right femurs were removed from the vials, dried with gauze, weighed, and placed in new vials containing deionized water. The bones were reweighed in the water. As previously described, using Archimedes' principle, bone density was calculated ( $\mathrm{g} / \mathrm{cm}^{3}$ bone volume) (23). 
Statistical analysis. In this study, the data are presented as the means \pm SEM. Statistical analysis was performed using the GraphPad Prism software (GraphPad Software, Inc., San Diego, CA, USA). One-way ANOVA was used to evaluate the treatment effect, followed by Dunnett's multiple comparison test. A p-value $<0.05$ was considered to indicate a statistically significant difference.

\section{Results}

LRC is not cytotoxic to RAW 264.7 cells. Prior to the in vitro tests, we measured the effects of LRC on cell viability. As shown in Fig. 1, all concentrations of LRC exerted equivalent effects on cell viability. LRC was not cytotoxic to RAW 264.7 cells. Thus, the following experiments were undertaken using a range of LRC concentrations (1,10 and $100 \mu \mathrm{g} / \mathrm{ml})$.

LRC inhibits osteoclastogenesis in RAW 264.7 cells induced by RANKL. To examine the effect of LRC on osteoclastogenesis, RAW 264.7 cells exposed to RANKL were stained using a TRAP staining kit. RAW 264.7 cells were stimulated with RANKL to differentiate into TRAP-positive cells, and we noted that $100 \mu \mathrm{g} / \mathrm{ml}$ LRC significantly decreased the

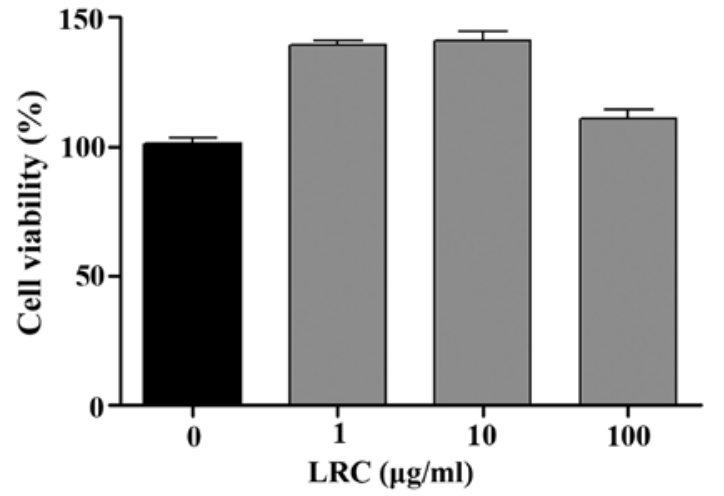

Figure 1. The effect of Lycii Radicis Cortex (LRC) on cell viability in RAW 264.7 cells. Data represent the means \pm SEM of three independent experiments.

number of TRAP-positive cells (Fig. 2A and B). Furthermore, as shown in Fig. 2C, it was also clear that LRC exerted an inhibitory effect on TRAP activity.

LRC inhibits pit formation. The effect of LRC on pit formation of mature osteoclasts was studied. As shown in Fig. 3A,

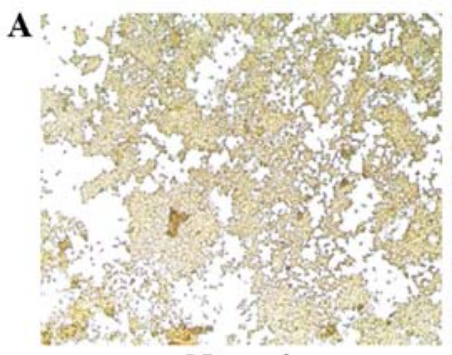

Normal

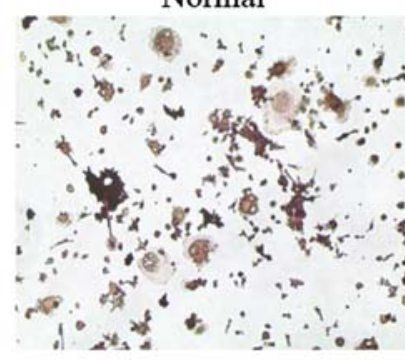

$\mathrm{LRC} 1 \mu \mathrm{g} / \mathrm{ml}$

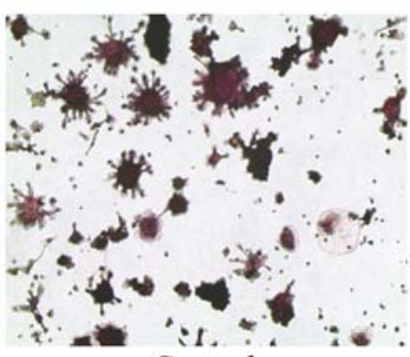

Control

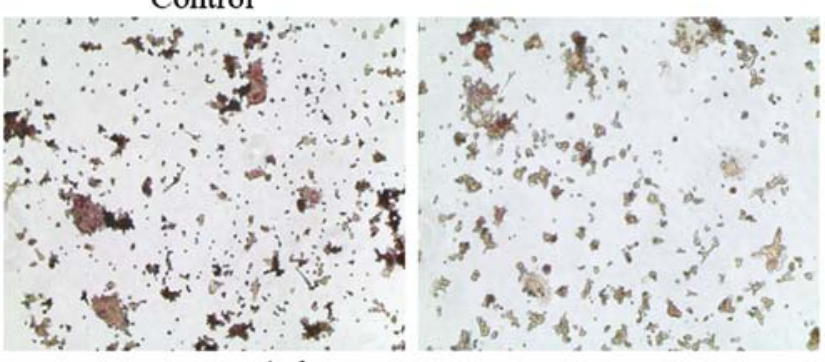

LRC $100 \mu \mathrm{g} / \mathrm{ml}$
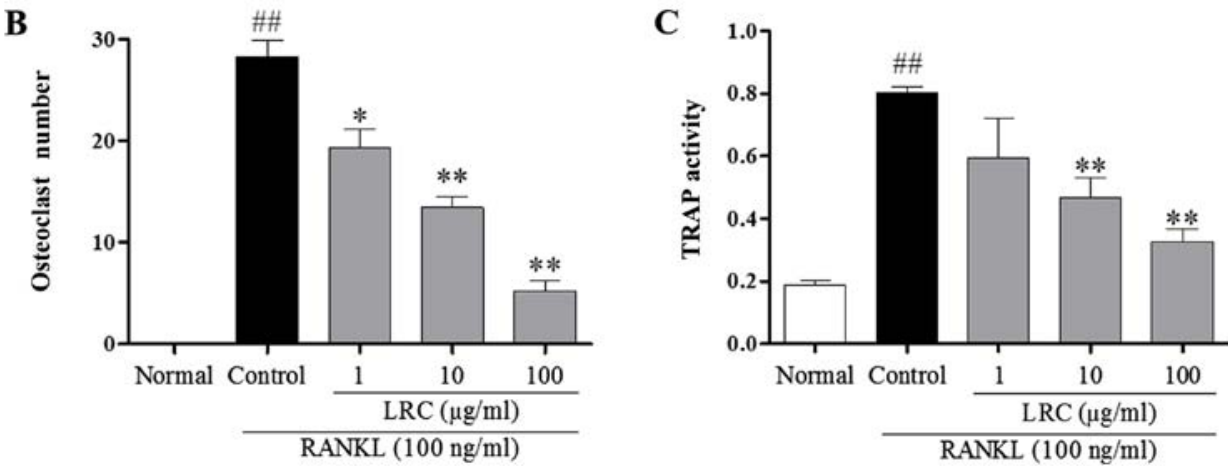

Figure 2. The effect of Lycii Radicis Cortex (LRC) on receptor activator of nuclear factor- $\kappa \mathrm{B}$ (NF- $\kappa \mathrm{B})$ ligand (RANKL)-induced osteoclast formation in RAW 264.7 cells. (A) Tartrate-resistant acid phosphatase (TRAP)-positive cells were captured using an inverted microscope (x100). (B) TRAP-positive cells were counted, and (C) TRAP activity was measured using an ELISA reader (optical density, $405 \mathrm{~nm}$ ). Data are represented as the means \pm SEM of three independent experiments. ${ }^{\# \#} \mathrm{p}<0.01$ compared with normal; ${ }^{* *} \mathrm{p}<0.01$ and ${ }^{*} \mathrm{p}<0.05$ compared with control. Normal, untreated cells. 

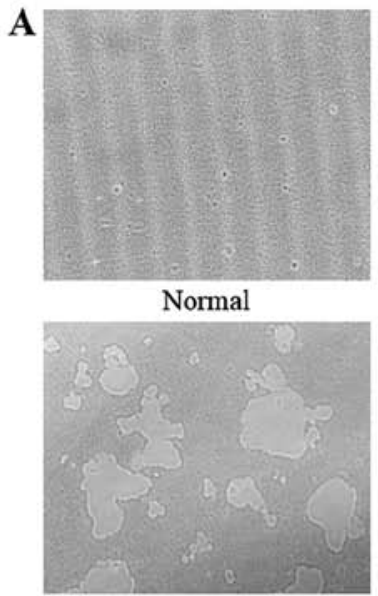

$\mathrm{LRC} 1 \mu \mathrm{g} / \mathrm{ml}$

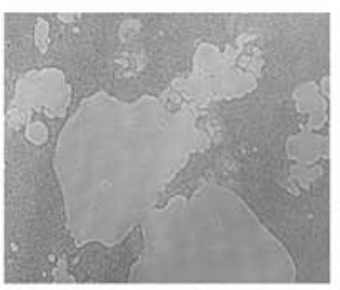

Control

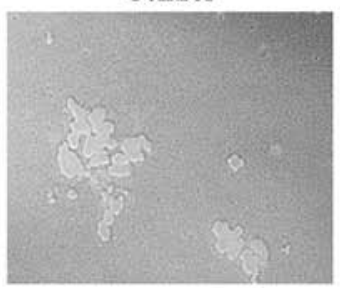

LRC $10 \mu \mathrm{g} / \mathrm{ml}$

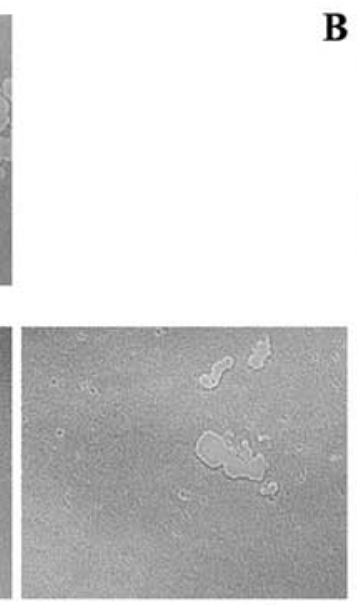

$\mathrm{LRC} 100 \mu \mathrm{g} / \mathrm{ml}$

B

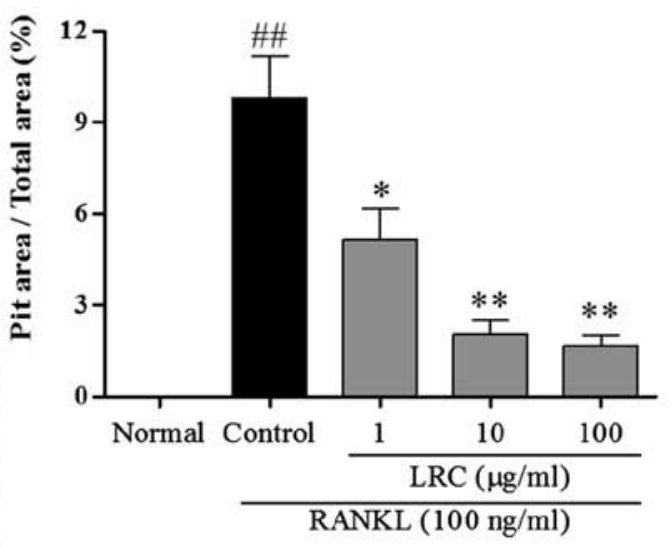

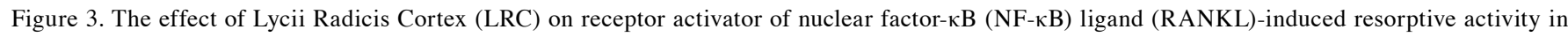
RAW 264.7 cells. (A) Pit area was observed under the inverted microscope (x100). (B) Pit area was measured using ImageJ software. Data are represented as the means \pm SEM of three independent experiments. ${ }^{\# \#} \mathrm{p}<0.01$ compared with normal; ${ }^{* *} \mathrm{p}<0.01$ and ${ }^{*} \mathrm{p}<0.05$ compared with control. Normal, untreated cells.

A

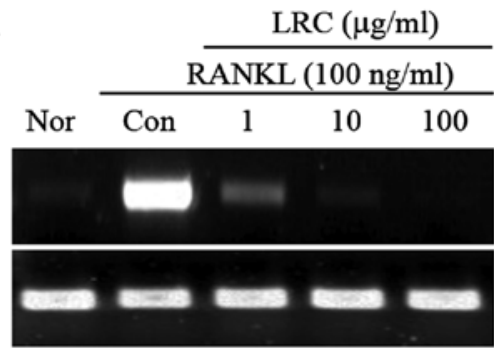

$\mathbf{C}$

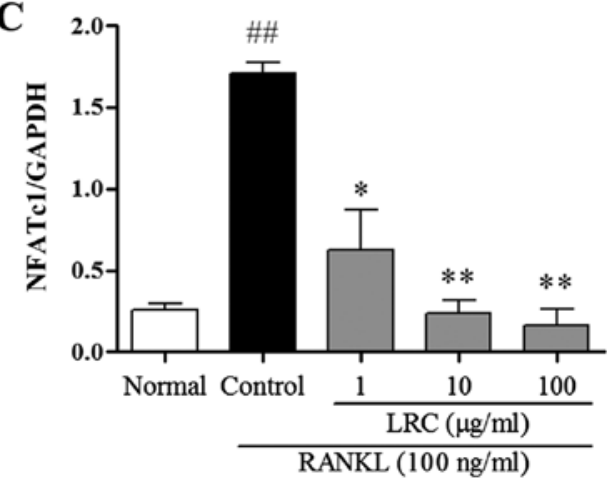

B

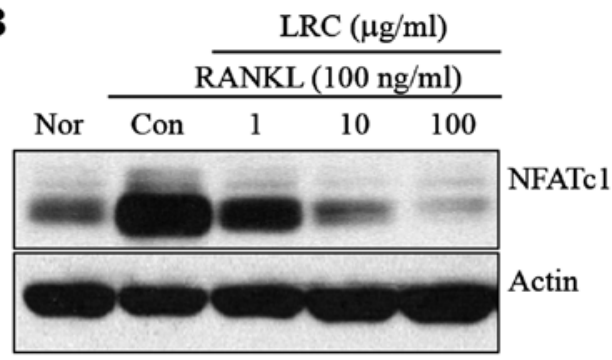

D

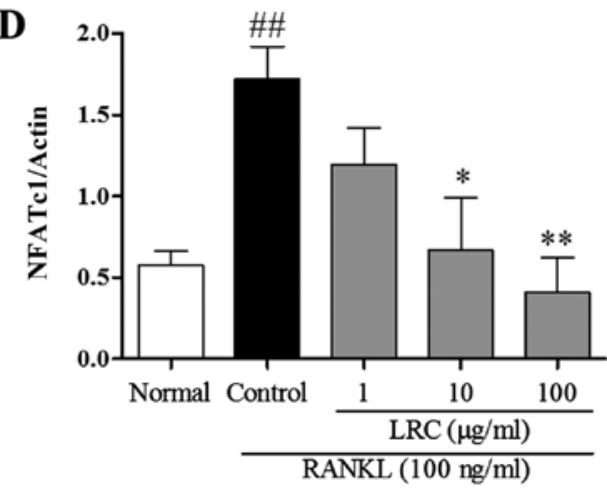

Figure 4. The effect of Lycii Radicis Cortex (LRC) on mRNA and protein expression of nuclear factor of activated T-cells cytoplasmic 1 (NFATc1) in receptor activator of nuclear factor- $\kappa \mathrm{B}(\mathrm{NF}-\kappa \mathrm{B})$ ligand (RANKL)-stimulated RAW 264.7 cells. (A) mRNA expression was detected using RT-qPCR. (B) The protein level was determined by western blot analysis. (C and D) mRNA and protein expression of NFATc1 were normalized to GAPDH and actin. Data are represented as the means \pm SEM of three independent experiments ${ }^{\# \#} \mathrm{p}<0.01$ compared with normal; ${ }^{* *} \mathrm{p}<0.01$ and ${ }^{*} \mathrm{p}<0.05$ compared with control. Nor (normal), untreated cells.

the area of resorption pit lacunae was significantly reduced by treatment with LRC. It was clear that the measured areas markedly decreased after treatment with LRC, in a dose-dependant manner (Fig. 3B).

LRC inhibits NFATcl expression. In order to confirm the inhibitory effect of LRC on osteoclastogenesis and bone resorption, we measured the expression of important osteoclast differentiation indicators, NFATc1. NFATc1 is known to be a master transcription factor in osteoclastogenesis (4). Thus, in the present study, mRNA and protein levels of NFATc1 were measured. As shown in Fig. 4, we noted that the levels were significantly upregulated upon exposure to RANKL, and LRC exerted a marked inhibitory effect on mRNA and protein expression levels. In addition, we noted that LRC did not markedly affect the expression of housekeeping genes such as GAPDH and actin.

LRC inhibits c-Fos expression. We measured the expression of c-Fos, which contributes to osteoclastogenesis via the downregulation of NFATc1. We measured the effect of LRC on c-Fos mRNA and protein levels. As shown in Fig. 5A, the 
A
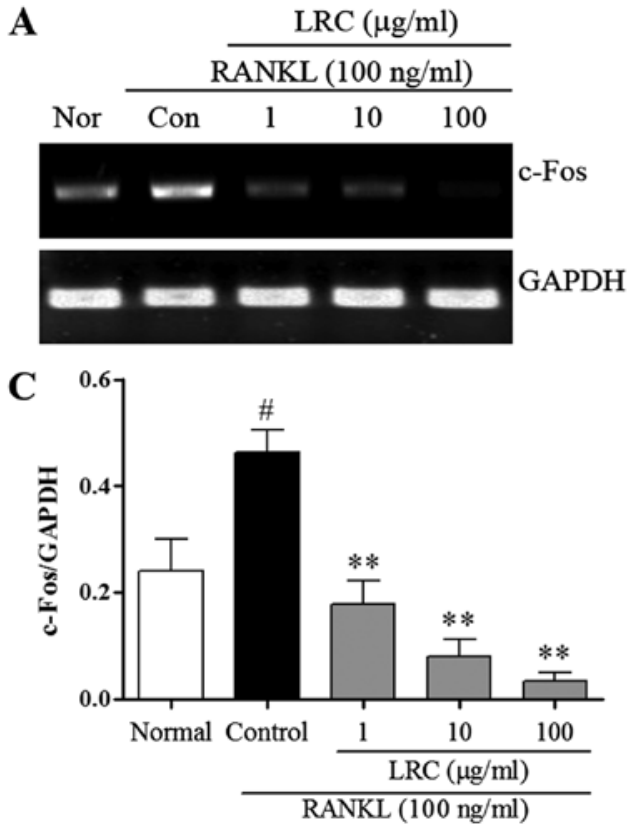

B

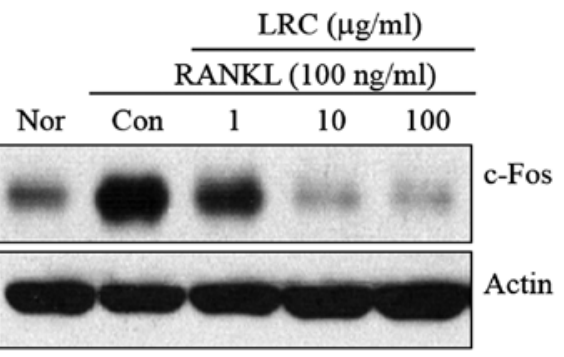

D

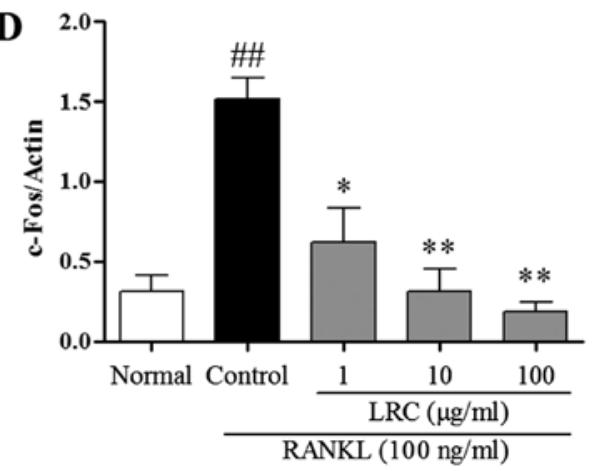

Figure 5. The effect of LRC on mRNA and protein expression of c-Fos in RANKL-stimulated RAW 264.7 cells. (A) mRNA expression was detected using RT-qPCR. (B) Protein levels were determined by western blot analysis. (C and D) mRNA and protein expression of c-Fos was normalized to GAPDH and actin. Data are represented as the means \pm SEM of three independent experiments ${ }^{\# \#} \mathrm{p}<0.01$ and ${ }^{\#} \mathrm{p}<0.05$ compared with normal; ${ }^{* *} \mathrm{p}<0.01$ and ${ }^{*} \mathrm{p}<0.05$ compared with control. Nor (normal), untreated cells.
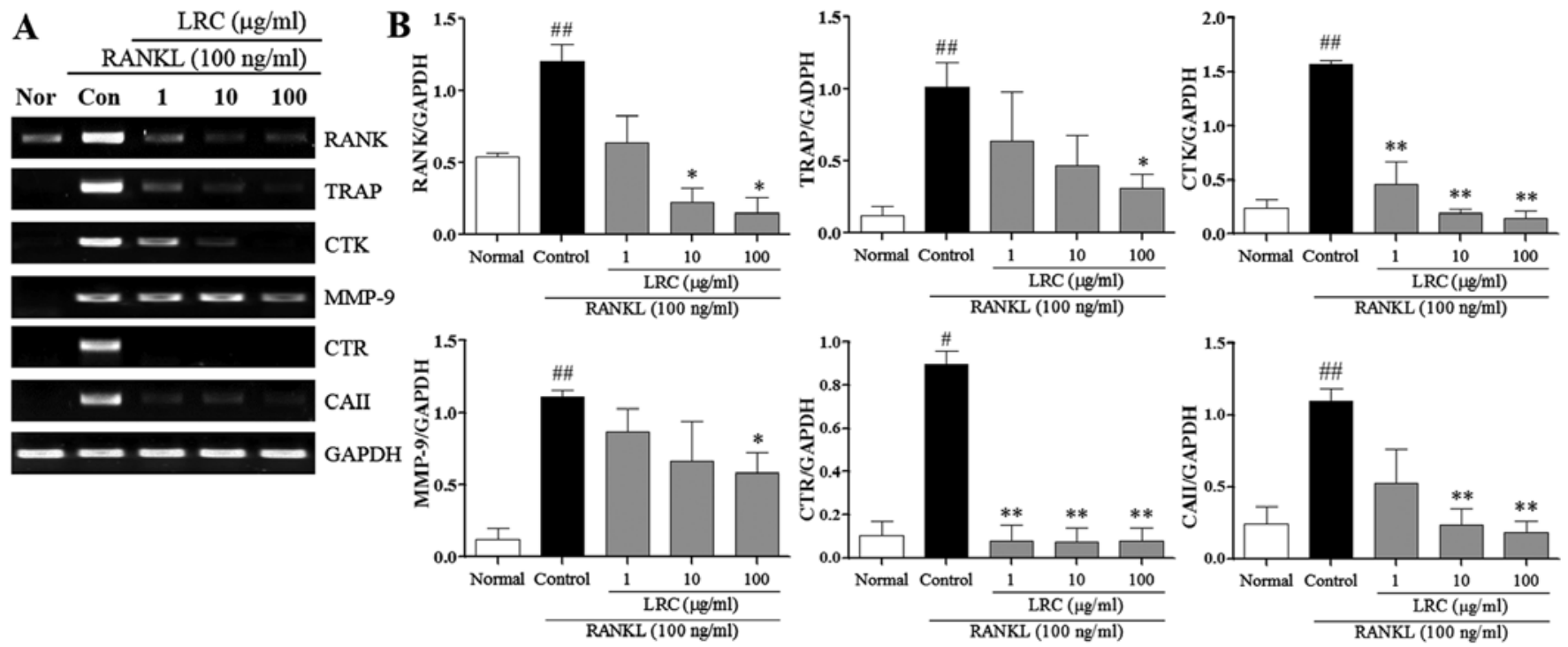

Figure 6. Effect of Lycii Radicis Cortex (LRC) on the expression of osteoclastogenesis-related genes in receptor activator of nuclear factor- $\mathrm{kB}$ (NF- $\mathrm{kB}$ ) ligand (RANKL)-stimulated RAW 264.7 cells. (A) mRNA expression was detected using RT-qPCR. (B) mRNA expression was normalized to GAPDH. Data are represented as the means \pm SEM of three independent experiments. ${ }^{\# \#} \mathrm{p}<0.01$ and ${ }^{*} \mathrm{p}<0.05$ compared with normal; ${ }^{* * *} \mathrm{p}<0.01$ and ${ }^{*} \mathrm{p}<0.05$ compared with control. Normal, untreated cells.

mRNA expression of c-Fos was induced slightly in normal cells, and in RAW 264.7 cells exposed to RANKL we noted significantly increased mRNA expression of c-Fos compared with normal cells. LRC exerted a marked inhibitory effect on mRNA expression. As shown in Fig. 5B, the protein levels of c-Fos were also significantly upregulated upon exposure to RANKL, whereas LRC clearly exerted a significant inhibitory effect on the protein levels. Moreover, we noted that LRC did not markedly affect the expression of the housekeeping genes GAPDH and actin.
LRC inhibits the expression of osteoclastogenesis-related genes. In the present study, we also examined the effect of LRC on osteoclastogenesis-related genes stimulated by RANKL, namely RANK, TRAP, CTK, MMP-9, CTR and carbonic anhydrase II (CAII). As shown in Fig. 6, the mRNA expression of RANK was induced, at a low level, in normal cells, and in RAW 264.7 cells exposed to RANKL we noted significantly increased mRNA expression of RANK compared with normal cells. LRC exerted significant inhibitory effects on RANK expression at concentrations of 10 and $100 \mu \mathrm{g} / \mathrm{ml}$. Moreover, 

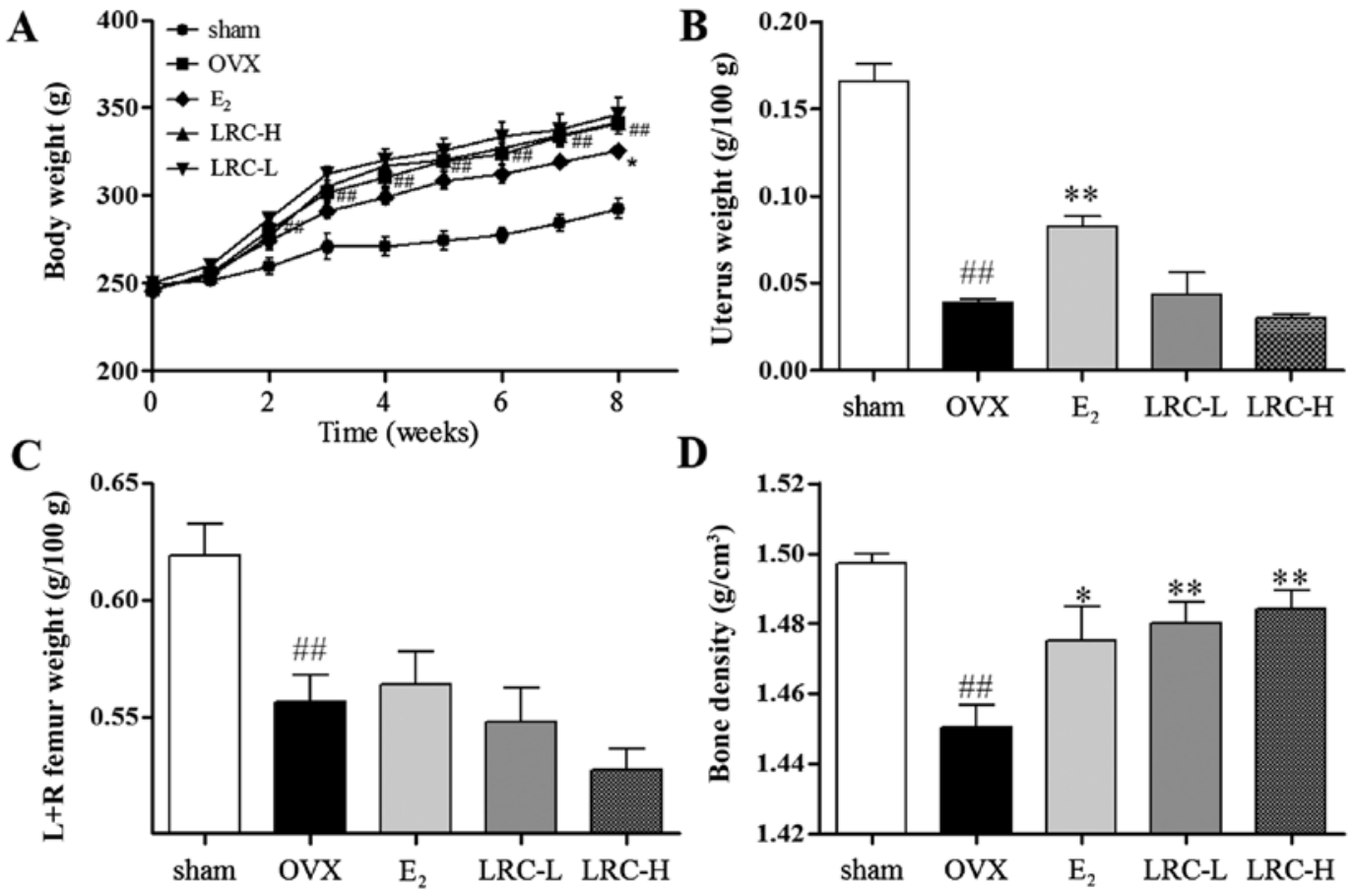

Figure 7. The effect of Lycii Radicis Cortex (LRC) on ovariectomized (OVX) rats. (A) Changes in body weight in rats which had undergone ovariectomy, (B) the rats were sacrificed and the uterus and (C) femurs from both sides were weighed. (D) Bone density of femurs was measured using Archimedes' principle. Data are represented as the means \pm SEM of eight independent experiments. ${ }^{\# \#}$ p $<0.01$ compared with sham-operated group; ${ }^{* *}$ p $<0.01$ and ${ }^{*}$ p $<0.05$ compared with OVX. LRC-L, rats treated with low doses of LRC; LRC-H, rats treated with high doses of LRC.

the mRNA expression of TRAP, CTK, MMP-9, CTR and CAII were significantly upregulated upon exposure to RANKL. LRC exerted a marked inhibitory effect on TRAP and MMP-9 expression at concentrations of $100 \mu \mathrm{g} / \mathrm{ml}$. In addition, we noted that LRC markedly reduced the expression of CTK and CTR at all concentrations and significantly inhibited the expression of CAII at concentrations of 10 and $100 \mu \mathrm{g} / \mathrm{ml}$.

LRC increases bone density in the OVX rat model. We aimed to investigate whether LRC prevents ovariectomy-induced bone loss. As shown in Fig. 7A, after the rats underwent the ovariectomy, the body weight of the OVX group significantly increased (as measured weekly) compared with the shamoperated group. In the LRC-treated groups, no significant changes in body weight were noted, and in the $\mathrm{E}_{2}$-treated group body weight was significantly inhibited compared with the OVX group. In the OVX group, a significant decrease in the weight of the uterus compared with the sham-operated group was noted (Fig. 7B). In the groups treated with low and high concentrations of LRC, no significant changes in uterus weight compared with the OVX group were noted. In the $\mathrm{E}_{2}$-treated group, a significant decrease in uterus weight loss compared with the OVX group was clear. We noted in the OVX group significantly decreased femur weight compared with the sham-operated group (Fig. 7C). In the groups treated with low and high concentrations of LRC and the $\mathrm{E}_{2}$-treated group, no significant changes in femur weight were noted compared with the OVX group. We also measured the bone density of the femurs using Archimedes' principle (Fig. 7D). The OVX group showed significantly decreased bone density compared with the sham-operated group. Moreover, in the LRC-treated groups, we noted a significant decrease in bone density loss in femurs compared with the OVX group. In the $\mathrm{E}_{2}$-treated group, we also noted decreased bone density loss compared with the OVX group. Thus, our results indicate that LRC decreased bone density loss without markedly affecting body weight, femur weight or uterine weight.

LRC exerts an inhibitory effect on trabecular area loss in OVX rats. To determine the in vivo effect of LRC on OVX-induced bone loss, we used histological staining on the femur samples. In the OVX group, we noted a significantly decreased trabecular area compared with the sham-operated group (Fig. 8B-F). In the $\mathrm{E}_{2}$-treated group the trabecular area loss was decreased compared with the OVX group. In the group treated with low concentrations of LRC, only a small decrease in trabecular area loss was noted compared with the OVX group. However, in the group treated with a high concentration of LRC a significant decrease in trabecular area loss compared with the OVX group was noted.

\section{Discussion}

In the present study, we demonstrated that LRC exerted an inhibitory effect on osteoclastogenesis through the reduction of key transcription factors such as NFATc1 and c-Fos. We noted that LRC also suppressed expression of osteoclastogenesis-related markers. Moreover, LRC inhibited bone loss in the OVX rat model. Abnormal bone resorption of osteoclasts is an important causal factor in osteoporosis, and as such, suppressing osteoclastogenesis is a significant step in osteoporosis treatment (6).

TRAPs are expressed particularly in osteoclasts and are commonly used as phenotype markers of osteoclasts; treat- 

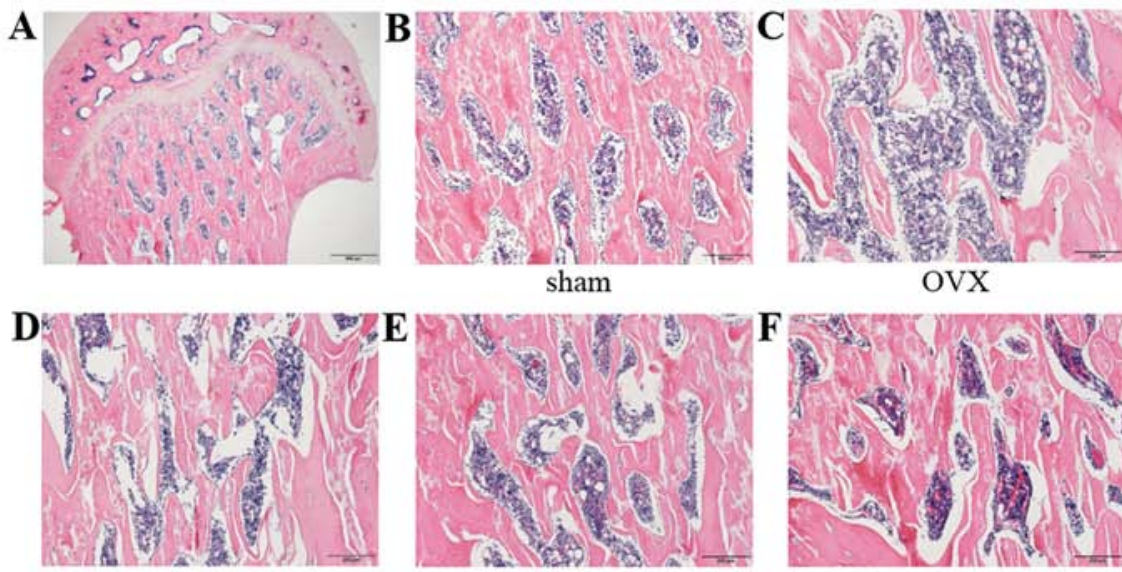

$\mathrm{E}_{2}$

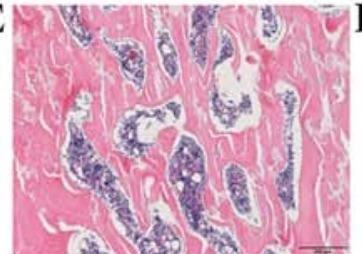

LRC-L

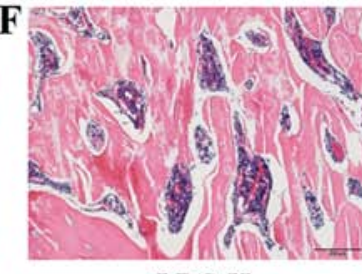

LRC-H
G

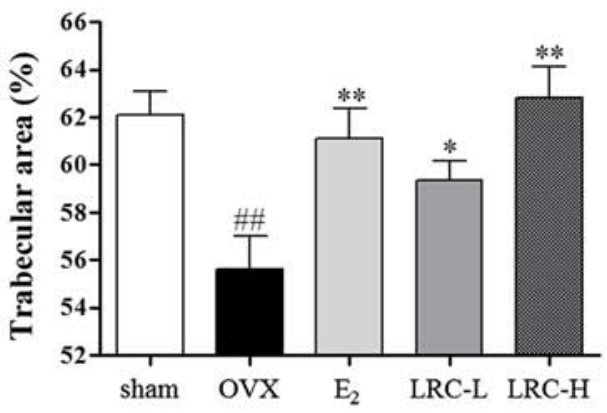

Figure 8. Effect of Lycii Radicis Cortex (LRC) on the trabecular area and bone loss in rats which underwent an ovariectomy; histological examination of tissue sections from the femoral heads of rats using H\&E staining. Femurs of each group were captured using a light microscope. (A) Representative femoral heads (histological staining). Scale bar, $500 \mu \mathrm{m}$. (B) Sham-operated rats, (C) ovariectomized (OVX) rats, (D) rats which were orally administered estradiol, (E) rats which were orally administered $5 \mathrm{mg} / \mathrm{kg} \mathrm{LRC} \mathrm{(LRC-L),} \mathrm{(F)} \mathrm{rats} \mathrm{which} \mathrm{were} \mathrm{orally} \mathrm{administered} 50 \mathrm{mg} / \mathrm{kg}$ LRC (LRC-H). Scale bar, 200 $\mu \mathrm{m}$. (G) Trabecular area was measured using ImageJ software. Data are represented as the means \pm SEM of eight independent experiments. ${ }^{\# \#<0.01 ~ c o m p a r e d ~ w i t h ~ s h a m-~}$ operated group; ${ }^{* *} \mathrm{p}<0.01$ and ${ }^{*} \mathrm{p}<0.05$ compared with OVX.

ment of RAW 264.7 cells with RANKL has been shown to easily induce cell differentiation into osteoclasts, which are TRAP-positive cells (24). The study of TRAP-positive cell formation and activity is a well-known method of determining osteoclast formation and function $(25,26)$. In the present study, we noted that LRC inhibited osteoclast formation and TRAP activity. These results indicate that LRC exerts an inhibitory effect on osteoclastogenesis.

Mature osteoclasts result in resorption lacunae and pit formation upon stimulation with RANKL $(27,28)$. Functionally, it is important that pit formation be used when identifying the osteoclast phenotype (24). In the present study, in order to investigate the effects of LRC on osteoclastic bone resorption, RAW 264.7 cells were cultured with RANKL. The density of pits was significantly reduced by LRC treatment. This suggests that LRC also exerted an inhibitory effect on mature osteoclast function.

In order to analyze molecular mechanisms, we measured osteoclastogenesis-related markers. In previous research it has been demonstrated that NFATc1 is an important transcription factor for RANKL-mediated osteoclast differentiation, fusion, and activation $(9,10)$. It has also been noted that overexpression of NFATc1 induces differentiation into osteoclasts even in cases of RANKL deficiency (29). Moreover, in NFATc1 knockout mice, defective osteoclast differentiation and osteopetrosis have been noted (30). In the present study, we noted that LRC exerted an inhibitory effect on NFATc1 mRNA and protein levels. NFATc1 plays an important role in osteoclast activation through the release of osteoclastogenesis-related genes such as TRAP, CTK, MMP-9 and CTR; the expression of TRAP, CTK and MMP-9 genes, which are the main markers responsible for the degradation of bone mineral and collagen matrices, are regulated by NFATc1 (4,10-12). Calcitonin suppresses both osteoclast formation and bone resorption and is a primary treatment for patients with hypercalcemia and increased bone turnover. The binding of calcitonin to its receptor has long been known to reduce osteoclast activation $(4,31)$. In the present study, we demonstrated that LRC inhibited expression of the
TRAP, CTK, MMP-9 and CTR genes. These data indicate that LRC inhibits osteoclastogenesis-related genes through suppression of NFATc1, which is an important transcription factor in osteoclastogenesis.

The interaction between RANK and RANKL is essential for osteoclast differentiation and activation. Binding of RANKL to RANK results in the recruitment of c-Fos, and subsequent stimulation of c-Fos results in the activation of NFATc1 $(9,10)$. The deletion of the gene-encoding c-Fos in mice leads to defective osteoclast differentiation and osteopetrosis (32). In the present study, LRC exerted inhibitory effects on c-Fos mRNA and protein levels. The data indicated that LRC inhibited osteoclastogenesis through suppression of NFATc1 following inhibition of c-Fos expression in LRC-treated cells. c-Fos has also previously been shown to regulate osteoclastogenesisrelated genes such as CAII. CAII influences bone resorption and osteoclast formation $(12,33)$. CAII affects the surface of the bone in an acid environment $(2,34)$. The acidic environment stimulates bone mineralization, and the demineralized organic ingredient of bone is resorbed by TRAP, CTK and MMP-9 $(2,4,35)$. In the present study, LRC inhibited the expression of CAII mRNA expression. These data indicate that LRC inhibits CAII through suppression of c-Fos. We also studied the expression of RANK, which regulates the expression of c-Fos. RANKL-RANK conjugation is important in the early stages of RAW 264.7 cell differentiation $(4,12)$. We showed that LRC inhibited RANK mRNA expression. The data demonstrated that LRC suppresses the expression of c-Fos by decreasing the signaling cascades connected with RANKL.

OVX is a widely used experimental method for inducing post-menopausal osteoporosis in females (22). Estrogen deficiency is accompanied by atrophy of organs such as the uterus (36), and atrophy of the uterus is evidence of the success of the ovariectomy. In the present study, OVX rats exhibited significantly reduced uterus weight, which was also observed in other studies, and in the $17 \beta$-estradiol treated group the uterus weight loss was reduced (37-39). However, in the LRC-treated 
groups no significant changes were noted in uterus weight In addition, OVX dramatically increased body weight. The mechanisms by which OVX induces an increase in body weight remain unclear; it seems that body fat accumulation is due to estrogen deficiency (40). However, in the present study we noted that LRC did not have a marked effect on body weight. The results suggest that the effect of LRC on OVX-induced rats does not conform to hormonal-related factors, but other factors.

Post-menopausal osteoporosis has been noted as being correlated with ovarian hormone deficiency following menopause, and can be induced by decreasing bone density, which increases bone resorption and deficient bone formation $(41,42)$. Reduced bone density is the main cause of fractures (43). Bones in OVX rats are also characterized by reduced bone density and reduced trabecular area (41). In the present study, LRC treatment exerted greater effects than $17 \beta$-estradiol. The data from our experiments demonstrate that LRC is a beneficial therapeutic agent which prevents bone loss due to post-menopausal osteoporosis.

In conclusion, the results of the present study suggest that LRC exerts an inhibitory effect on menopausal osteoporosis. LRC reduced the expression of osteoclastogenesis-related markers NFATc1, c-Fos, RANK, TRAP, CTK, CTR, MMP-9 and CAII and reduced bone density loss and trabecular area loss in the OVX rat model. In terms of bone density loss and trabecular area loss, levels in rats treated with high doses of LRC in particular were notably higher than in the $E_{2}$-treated groups. However, it is possible that LRC has side-effects and this aspect requires additional study.

\section{References}

1. Burge R, Dawson-Hughes B, Solomon DH, Wong JB, King A and Tosteson A: Incidence and economic burden of osteoporosisrelated fractures in the United States, 2005-2025. J Bone Miner Res 22: 465-475, 2007.

2. Teitelbaum SL: Bone resorption by osteoclasts. Science 289: 1504-1508, 2000.

3. Rodan GA and Martin TJ: Therapeutic approaches to bone diseases. Science 289: 1508-1514, 2000.

4. Boyle WJ, Simonet WS and Lacey DL: Osteoclast differentiation and activation. Nature 423: 337-342, 2003.

5. Wensel TM, Iranikhah MM and Wilborn TW: Effects of denosumab on bone mineral density and bone turnover in postmenopausal women. Pharmacotherapy 31: 510-523, 2011.

6. de Villiers TJ: Bone health and osteoporosis in postmenopausal women. Best Pract Res Clin Obstet Gynaecol 23: 73-85, 2009.

7. Collin-Osdoby P and Osdoby P: RANKL-mediated osteoclast formation from murine RAW 264.7 cells. Methods Mol Biol 816: 187-202, 2012

8. Suda T, Takahashi N, Udagawa N, Jimi E, Gillespie MT and Martin TJ: Modulation of osteoclast differentiation and function by the new members of the tumor necrosis factor receptor and ligand families. Endocr Rev 20: 345-357, 1999.

9. Grigoriadis AE, Wang ZQ, Cecchini MG, Hofstetter W, Felix R, Fleisch HA and Wagner EF: c-Fos: a key regulator of osteoclastmacrophage lineage determination and bone remodeling. Science 266: 443-448, 1994

10. Zhao Q, Wang X, Liu Y, He A and Jia R: NFATc1: functions in osteoclasts. Int J Biochem Cell Biol 42: 576-579, 2010

11. Choi HJ, Park YR, Nepal M, Choi BY, Cho NP, Choi SH, Heo SR, Kim HS, Yang MS and Soh Y: Inhibition of osteoclastogenic differentiation by Ikarisoside A in RAW 264.7 cells via JNK and NF-kappaB signaling pathways. Eur J Pharmacol 636: 28-35, 2010.

12. Fujisaki K, Tanabe N, Suzuki N, Kawato T, Takeichi O, Tsuzukibashi O, Makimura M, Ito K and Maeno M: Receptor activator of NF-kappaB ligand induces the expression of carbonic anhydrase II, cathepsin K, and matrix metalloproteinase-9 in osteoclast precursor RAW264.7 cells. Life Sci 80: 1311-1318, 2007.
13. Wimalawansa SJ: Prevention and treatment of osteoporosis: efficacy of combination of hormone replacement therapy with other antiresorptive agents. J Clin Densitom 3: 187-201, 2000.

14. Alexandersen P, Toussaint A, Christiansen C, Devogelaer JP, Roux C, Fechtenbaum J, Gennari C, Reginster JY and Ipriflavone Multicenter European Fracture S; Ipriflavone Multicenter European Fracture Study: Ipriflavone in the treatment of postmenopausal osteoporosis: a randomized controlled trial. JAMA 285: 1482-1488, 2001.

15. Herbal Pharmacology Compilation Committee: Herbal Pharmacology. Shinil books, Seoul, pp715-717, 2010.

16. Herbology Editorial Committee of Korean Medicine schools: Boncho-hak [Herbology]. Younglimsa, Seoul, pp534-535, 2004.

17. Song MY, Jung HW, Kang SY, Kim KH and Park YK: Antiinflammatory effect of Lycii radicis in LPS-stimulated RAW 264.7 macrophages. Am J Chin Med 42: 891-904, 2014.

18. Cho SH, Park EJ, Kim EO and Choi SW: Study on the hypochlolesterolemic and antioxidative effects of tyramine derivatives from the root bark of Lycium chenese Miller. Nutr Res Pract 5: 412-420, 2011.

19. Kim SJ, Lee L, Kim JH, Lee TH and Shim I: Antidepressant-like effects of lycii radicis cortex and betaine in the forced swimming test in rats. Biomol Ther (Seoul) 21: 79-83, 2013.

20. Jeong JC, Kim SJ, Kim YK, Kwon CH and Kim KH: Lycii cortex radicis extract inhibits glioma tumor growth in vitro and in vivo through downregulation of the Akt/ERK pathway. Oncol Rep 27: 1467-1474, 2012.

21. Gao D, Li Q, Liu Z, Li Y, Liu Z, Fan Y, Li K, Han Z and Li J: Hypoglycemic effects and mechanisms of action of Cortex Lycii Radicis on alloxan-induced diabetic mice. Yakugaku Zasshi 127: 1715-1721, 2007.

22. Kalu DN: The ovariectomized rat model of postmenopausal bone loss. Bone Miner 15: 175-191, 1991.

23. Arjmandi BH, Alekel L, Hollis BW, Amin D, StacewiczSapuntzakis M, Guo P and Kukreja SC: Dietary soybean protein prevents bone loss in an ovariectomized rat model of osteoporosis. J Nutr 126: 161-167, 1996.

24. Hsu H, Lacey DL, Dunstan CR, Solovyev I, Colombero A, Timms E, Tan HL, Elliott G, Kelley MJ, Sarosi I, et al: Tumor necrosis factor receptor family member RANK mediates osteoclast differentiation and activation induced by osteoprotegerin ligand. Proc Natl Acad Sci USA 96: 3540-3545, 1999.

25. Tanaka H, Tanabe N, Shoji M, Suzuki N, Katono T, Sato S, Motohashi M and Maeno M: Nicotine and lipopolysaccharide stimulate the formation of osteoclast-like cells by increasing macrophage colony-stimulating factor and prostaglandin E2 production by osteoblasts. Life Sci 78: 1733-1740, 2006.

26. Tanabe N, Maeno M, Suzuki N, Fujisaki K, Tanaka H, Ogiso B and Ito K: IL-1 alpha stimulates the formation of osteoclast-like cells by increasing M-CSF and $\mathrm{PGE}_{2}$ production and decreasing OPG production by osteoblasts. Life Sci 77: 615-626, 2005.

27. Kim SN, Kim MH, Min YK and Kim SH: Licochalcone A inhibits the formation and bone resorptive activity of osteoclasts. Cell Biol Int 32: 1064-1072, 2008.

28. Jun AY, Kim HJ, Park KK, Son KH, Lee DH, Woo MH, Kim YS, Lee SK and Chung WY: Extract of Magnoliae Flos inhibits ovariectomy-induced osteoporosis by blocking osteoclastogenesis and reducing osteoclast-mediated bone resorption. Fitoterapia 83: 1523-1531, 2012.

29. Kim K, Lee SH, Ha Kim J, Choi Y and Kim N: NFATc1 induces osteoclast fusion via up-regulation of Atp6v0d2 and the dendritic cell-specific transmembrane protein (DC-STAMP). Mol Endocrinol 22: 176-185, 2008.

30. Winslow MM, Pan M, Starbuck M, Gallo EM, Deng L, Karsenty $G$ and Crabtree GR: Calcineurin/NFAT signaling in osteoblasts regulates bone mass. Dev Cell 10: 771-782, 2006.

31. Takahashi S, Goldring S, Katz M, Hilsenbeck S, Williams R and Roodman GD: Downregulation of calcitonin receptor mRNA expression by calcitonin during human osteoclast-like cell differentiation. J Clin Invest 95: 167-171, 1995.

32. Wang ZQ, Ovitt C, Grigoriadis AE, Möhle-Steinlein U, Rüther U and Wagner EF: Bone and haematopoietic defects in mice lacking c-fos. Nature 360: 741-745, 1992.

33. David JP, Rincon M, Neff L, Horne WC and Baron R: Carbonic anhydrase II is an AP-1 target gene in osteoclasts. J Cell Physiol 188: 89-97, 2001.

34. Sundaram K, Nishimura R, Senn J, Youssef RF, London SD and Reddy SV: RANK ligand signaling modulates the matrix metalloproteinase-9 gene expression during osteoclast differentiation. Exp Cell Res 313: 168-178, 2007. 
35. Andersen TL, del Carmen Ovejero M, Kirkegaard T, Lenhard T, Foged NT and Delaissé JM: A scrutiny of matrix metalloproteinases in osteoclasts: evidence for heterogeneity and for the presence of MMPs synthesized by other cells. Bone 35: 1107-1119, 2004.

36. Versi E, Harvey MA, Cardozo L, Brincat M and Studd JW: Urogenital prolapse and atrophy at menopause: a prevalence study. Int Urogynecol J Pelvic Floor Dysfunct 12: 107-110, 2001

37. Lim DW, Kim JG, Lee Y, Cha SH and Kim YT: Preventive effects of Eleutherococcus senticosus bark extract in OVX-induced osteoporosis in rats. Molecules 18: 7998-8008, 2013.

38. Lim DW and Kim YT: Dried root of Rehmannia glutinosa prevents bone loss in ovariectomized rats. Molecules 18: 5804-5813, 2013.

39. Hidaka S, Okamoto Y, Nakajima K, Suekawa M and Liu SY: Preventive effects of traditional Chinese (Kampo) medicines on experimental osteoporosis induced by ovariectomy in rats. Calcif Tissue Int 61: 239-246, 1997.

40. Dang ZC, van Bezooijen RL, Karperien M, Papapoulos SE and Löwik CW: Exposure of KS483 cells to estrogen enhances osteogenesis and inhibits adipogenesis. J Bone Miner Res 17: 394-405, 2002.
41. Wronski TJ, Lowry PL, Walsh CC and Ignaszewski LA: Skeletal alterations in ovariectomized rats. Calcif Tissue Int 37: 324-328, 1985.

42. Kalu DN, Liu CC, Salerno E, Hollis B, Echon R and Ray M: Skeletal response of ovariectomized rats to low and high doses of 17 beta-estradiol. Bone Miner 14: 175-187, 1991.

43. Hui SL, Slemenda CW and Johnston CC Jr: Baseline measurement of bone mass predicts fracture in white women. Ann Intern Med 111: 355-361, 1989. 